\title{
Erste Gehversuche einer ,Geschichtsschreibung von unten'
}

Immer wieder spürte ich bei meinen Gesprächen mit den DOMiD-Pionier*innen, wie prägend und identitätsstiftend ihre Erfahrungen als politische Flüchtlinge gewesen waren. Aber auch mit der Geschichte der Gastarbeiter*innen schienen sie von Beginn an eng verflochten: Aytaç Eryılmaz hatte mir erzählt, wie er sich bei seiner Flucht aus der Türkei als Gastarbeiter ausgegeben hatte. Ahmet Sezer war nach Deutschland gekommen, weil seine Schwester bereits als Gastarbeiterin in Deutschland lebte, und Tayfun Demir nahm sich der sogenannten Gastarbeiterliteratur an. Wie aber genau war diese Idee entstanden, in der Ruhrmetropole Essen ein Archiv zu gründen, das sich konsequent eben dieser Geschichte widmen sollte?

Das DOMiD-Archiv hatte Vorläufer, wie Türkei-Data, eine Initiative, die bereits seit 1987/88 existierte. Es handelte sich um einen Zusammenschluss von türkischen und deutschen Intellektuellen, Aytaç Eryılmaz hatte Türkei-Data zusammen mit der in Istanbul promovierten Diplom-Pädagogin Angela Eberding in Essen gegründet.

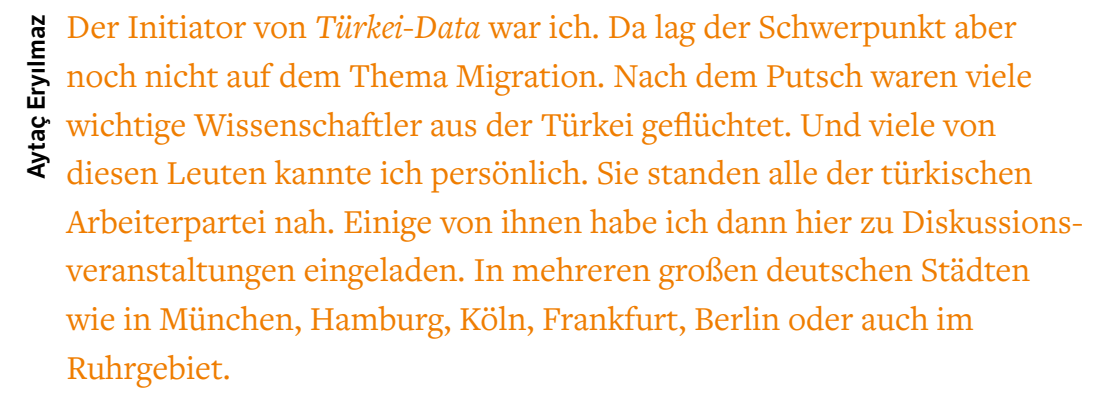

Zunächst hatte die Aufmerksamkeit von Türkei-Data der Dokumentation deutsch-türkischer Beziehungen im Allgemeinen gegolten. Mit vollständigem Namen hieß die Formation Türkei-Data e.V. Dokumentations- und Datenzentrum für wissenschaftliche Forschung über die Türkei e.V. Türkei-Data lud Referent*innen ein, die zu deutsch-türkischen Beziehungen zur Zeit des deutschen Kaiserreiches und des Osmanischen Reiches referierten oder zu Fragen einer Mitgliedschaft der Türkei in der Europäi- 


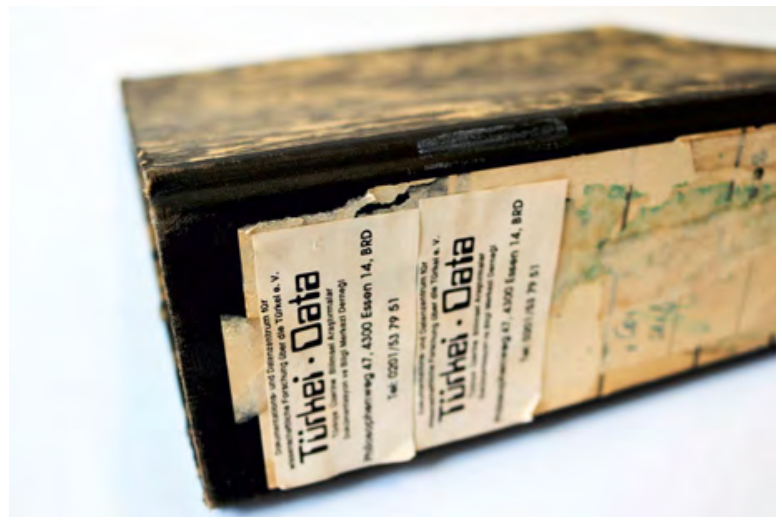

schen Union. ${ }^{1}$ In der Satzung, formuliert am 13. Mai 1988, heißt es, der Verein Türkei-Data bezwecke „die Förderung der wissenschaftlichen Forschung über die Türkei auf allen Gebieten, insbesondere über ihre Beziehungen zur Europäischen Gemeinschaft. Für diesen Zweck sammelt und klassifiziert er wissenschaftliche Literatur, Dokumente und Daten; knüpft er Kontakte mit Studenten, Wissenschaftlern und Hochschullehrern; nimmt er Beziehungen zu Hochschulen, Instituten, Stiftungen und Forschungseinrichtungen auf; organisiert er Konferenzen, Foren, Seminare und wissenschaftliche Gespräche; veröffentlicht er Bulletins, Bibliographien und wissenschaftliche Arbeiten. Er bezweckt die Förderung des Friedens, der Völkerverständigung und des demokratischen Geistes.“2 Um im letzten Passus die Fragestellungen der Gastarbeiter*innen-Migration erst anzudeuten: „Für diesen Zweck unterstützt er jegliche Tätigkeit zur Förderung der sozialen Integration der ausländischen Arbeitsmigranten und ihrer Familien in der Bundesrepublik Deutschland. Er trägt insbesondere zur Förderung der Erziehung, Aus- und Weiterbildung der ausländischen Arbeiter bei.“"

Auch das Türkei-Archiv in Berlin gehört zu DOMiDs Vorläuferorganisationen, dessen Gründer*innen sich ebenfalls linken, emanzipatorischen Ideen verpflichtet fühlten. Bereits zu Beginn der 1980er-Jahre wollten auch sie einen Beitrag zum Aufbau eines historischen Gedächtnisses der deutsch-türkischen Beziehungen leisten. Ihre Programmatik beschrieben sie selbst als Doppelstrategie: „Am 12.12.1981 wurde in Berlin das TürkeiArchiv gegründet. Ein Zweck dieser Gründung war es, das in der Bun- 
4 VA 0546 (8).

5 Ebd.

7 Hans-Günter Kleff, Vom Bauern zum Industriearbeiter: Zur kollektiven Lebensgeschichte der Arbeitsmigranten aus der Türkei, Mainz 1985. desrepublik und in West-Berlin vorhandene Material aus und über die Türkei zu sammeln und öffentlich zugänglich zu machen. Angesichts der damaligen Lage nach dem Militärputsch sollte allerdings auch versucht werden, in der Türkei gefährdetes Archivmaterial sozialer Bewegungen in Sicherheit zu bringen. “" Hier ist es der Gedanke der Sammlung, der bereits vorgedacht wird. „Das zweite Aktionsfeld des Türkei-Archivs sollte das Zusammenfassen und Öffentlichmachen vorhandener Privatarchive über die Migration zwischen der Türkei und Westeuropa sein. “5 Das große Vorbild des Türkei-Archivs in Berlin hinsichtlich seiner Konzeption und Wirkung war das damals in Berlin ansässige Forschungs- und Dokumentationszentrum Chile-Lateinamerika (FDCL). Doch gegen Ende 1986 waren die Bemühungen der Initiator*innen eingeschlafen, das Türkei-Archiv in Berlin zu verwirklichen. Tayfun Demirs Vorschlag, das Archiv nach Duisburg zu verlagern und dort im Ruhrgebiet einen Neuanfang zu versuchen, wurde deshalb im Mai 1987 aufgegriffen. „Wir hoffen sehr, dass das geplante Dokumentations- und Forschungszentrum für die Migranten aus der Türkei das wird, was das Türkei-Archiv nie schaffen konnte“, heißt es in dem Glückwunschschreiben des Migrationsforschers Hans-Günter-Kleff an die DOMiD-Gründer*innen. ${ }^{6}$

言

Seit 1979 war ich bei der Duisburger Stadtbibliothek. Aber in der Zeit fuhr ich immer wieder auch nach Berlin. Dort habe ich Dr. Arif Çağlar kennengelernt, einen Philosophen, der aber auch schon damals programmieren konnte. Und einen Architekten, Cihan Arın, der heute sehr bekannt ist in Berlin. Dann gehörte noch Günter Kleff zu der Gruppe, der das berühmte Buch „Vom Bauern zum Industriearbeiter“ geschrieben hat.7 Die hatten seit Anfang der 1980er-Jahre gemeinsam dieses Türkei-Archiv entwickelt. Hatten den SPIEGEL und den STERN von Anfang der 1950er- bis Anfang der 1980er-Jahre durchgesehen und alles fotokopiert, was darin über Gastarbeiter geschrieben worden war. Dann wollten sie nicht mehr. Und ich dachte, warum hole ich diese Kisten nicht nach Duisburg, und diese Idee. Diese vier Ordner kamen aus Berlin, das war die erste Überlieferung. 
In der Hohestraße hatte ich an der Tür ein Schild, TürkeiArchiv e.V. Aber dann geriet ich in eine Sackgasse, es ging nicht weiter. Ich hatte die Verantwortung für das Material, und ich wusste nicht wohin damit. Abends habe ich es immer wieder durchgeblättert, das zerfallene Zeitungspapier wieder auf Papier aufgeklebt, und mich gefragt: Was ist das überhaupt wert? Und was sollte man sammeln? Dann kam die Idee auf, diese ganzen verstreuten Zeugnisse zu sammeln, Hochzeitseinladungen, Einladungen zu Beschneidungsfesten oder zum Ersten Mai. Dazu die sogenannte ,graue Literatur ${ }^{8}$, die in diversen Zeitschriften veröffentlicht, aber sonst nirgendwo erfasst wird und verschwindet. So haben wir angefangen: die Drucksachen einfach gelocht und in Ordner gepackt. Heute würde das keiner mehr so machen. Aber das zeigt zumindest das Bewusstsein: Etwas drohte verloren zu gehen. Und wir wollten etwas dagegen unternehmen.

Sie haben etwas dagegen unternommen. Diese ,ersten Überlieferungen“ 8 Unter, grauer Literatur' versteht man in der Bibliothekswissenschaft kleine Auflagen verstreuter Schriften, die nicht über das offizielle Verlagswesen veröffentlicht und ohne kommerzielles Interesse im Privatdruck liegen heute im DOMiD-Archiv. Doch es brauchte noch fast zwei Jahre intensiver Diskussion, ehe sich die Vorläuferorganisationen Türkei-Data und Türkei-Archiv im Jahr 1990 endgültig auflösten und zum Verein DoMiT Dokumentationszentrum und Museum über die Migration aus der Türkei e.V. verschmolzen. 


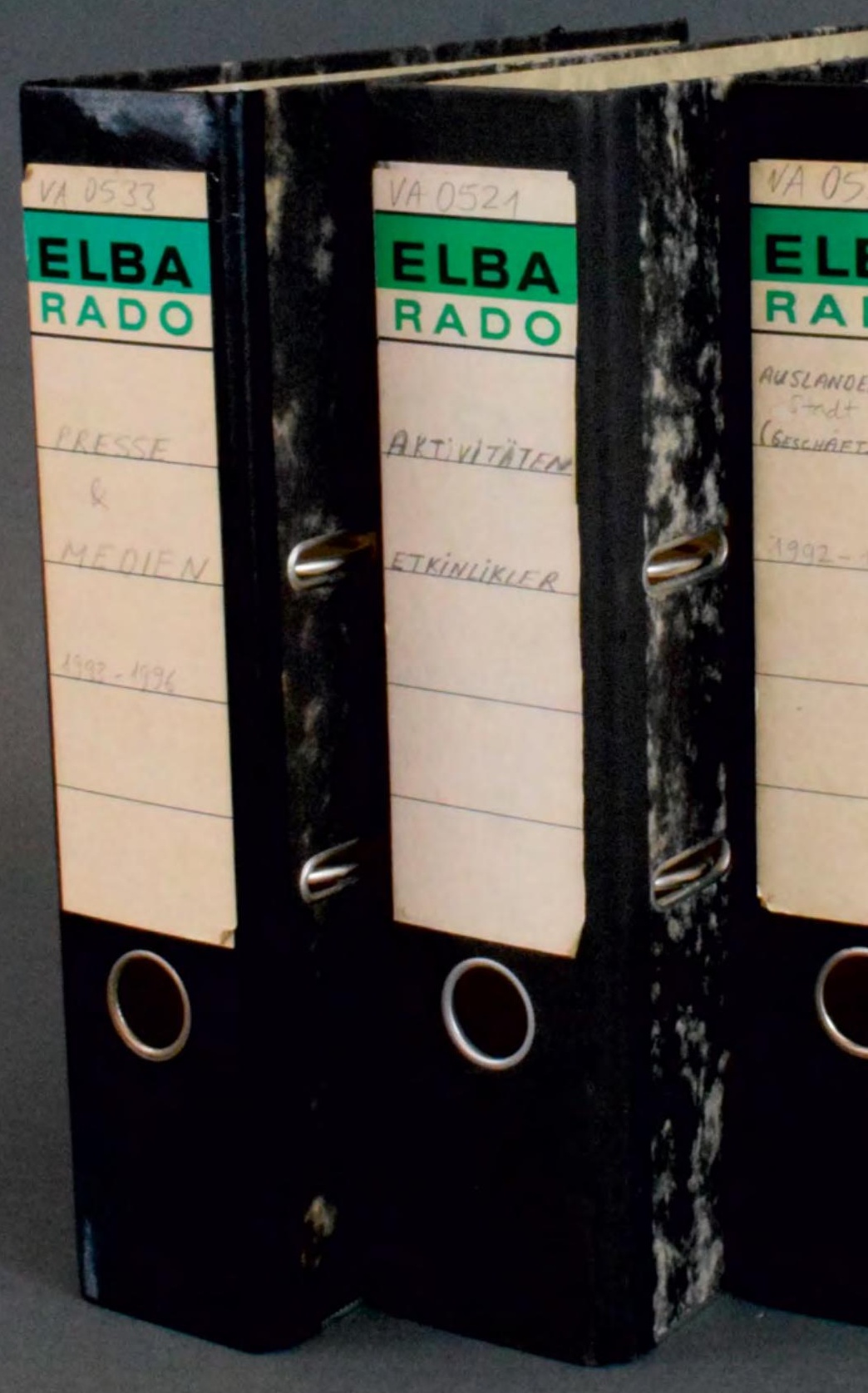




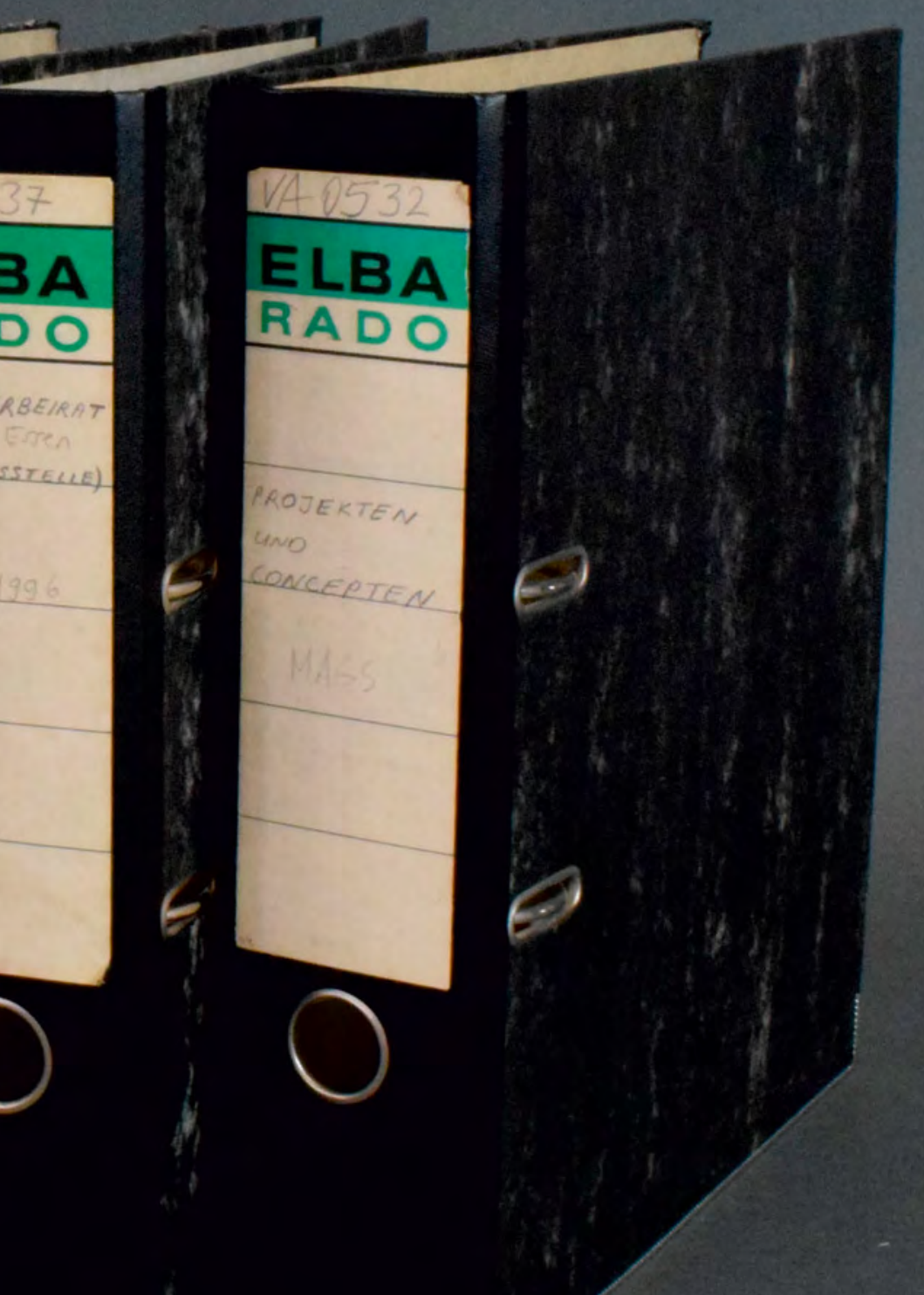

VA 0521, 0532, 0533, 0537. Lieblingsobjekt Tayfun Demir. DOMiD-Archiv, Köln

Zur frühesten Überlieferung der Vereinsgeschichte von DOMiD gehören Ordner von Anfang der 1990er-Jahre, gefüllt mit Presseartikeln zu diversen Migrationsthemen, dem Briefverkehr mit verschiedenen Unterstützer*innenorganisationen, Rechenschaftsberichten von frühen Ausstellungsaktivitäten sowie Konzeptpapieren u. v. m. 